\section{DISLIPIDEMIAS EM MULHERES FEIRANTES EM CIDADE NO SEMIÁRIDO BAIANO}

\section{DYSLIPIDEMIAS IN FEMALE MARKET WORKERS IN A CITY OF SEMIARID REGION OF BAHIA STATE}

Beatriz de Almeida Marques ${ }^{1}$ / Deíze Carvalho Pereira ${ }^{1}$ / Grasielle da Silva Santos ${ }^{1, *} /$ Marcela Andrade Rios ${ }^{1} /$ Raissa Neyla da Silva Domingues Nogueira ${ }^{1} /$ Rúbia Pinto Carvalho

\section{INTRODUÇÃO}

O trabalho caracteriza-se como uma condição preponderante para a realização humana e perfaça para além de objetivar somente a manutenção financeira do indivíduo, uma vez que possibilita ação transformadora sobre a natureza e si mesmo e exterioriza a capacidade criativa através do ofício que lhe é incumbido.

Em virtude da estagnação de proteção das leis sociais e trabalhistas, das transformações do capitalismo contemporâneo e aumento do desemprego estrutural, a economia informal brasileira tem-se desenvolvido rapidamente nos últimos anos.O setor informal caracteriza-se, principalmente, pela inserção do trabalhador de forma precária no mercado de trabalho (ILO, 2018).

$\mathrm{O}$ crescimento de trabalhos informais em meio ao assalariamento é um fenômeno mundial, visto que mais de $61 \%$ da população empregada no mundo está na economia informal. No continente africano $85,8 \%$ do emprego é informal, na Ásia e no Pacífico essa proporção é de 68,2\%, já nas Américas o índice chega a 40\%.No Brasil, o índice de informalidade econômica é de $46 \%$, sendo maior entre os homens $(37 \%)$, do que entre as mulheres $(21,5 \%)$ no setor informal (ILO, 2018).

Contudo, paulatinamente, os espaços sociais que antes eram preenchidos majoritariamente pela população masculina, passam por um processo de transformação cultural e comportamental, e no âmbito do trabalho no setor informal este remodelamento não se difere.

\section{RESUMO}

Objetivo: Descrever a prevalência de dislipidemias em trabalhadoras feirantes que atuam no mercado municipal de Guanambi/BA. Materiais e Métodos: Trata-se de um estudo epidemiológico de corte transversal e censitário, no qual foram analisados dados sociodemográficos, laborais e condições de saúde de trabalhadoras feirantes com dislipidemia. Os dados foram coletados por meio de questionários semiestruturados, realização de exames de sangue e avaliação antropométrica. Após coletados, os dados foram tabulados e analisados por meio do programa Epi Info, versão 7.0. Resultados: O estudo contou com uma população total de 426 trabalhadores, destes 241 eram do sexo feminino, 199 realizaram exame de sangue, sendo que, todas apresentaram algum tipo de dislipidemia, possuíam faixa etária entre 45 a 59 anos, ensino fundamental incompleto, a maioria possuía um parceiro (a) fixo. Grande parte não fazia uso de bebida alcoólica, nem de tabaco e não praticavam atividade física. As feirantes atuavam principalmente no pavilhão 3, possuíam jornada de trabalho semanal com mais de 44 horas e a maior parte relatou não apresentar diagnóstico médico para nenhuma de doença. Conclusão: Diante do exposto faz-se necessário que órgãos e instituições municipais desenvolvam políticas intervencionistas para garantir condições trabalhistas adequadas e ações educativas em saúde.

Palavras-chave: Saúde do Trabalhador. Trabalhadores Informais. Saúde da Mulher.

\section{ABSTRACT}

Objective: Describe the prevalence of dyslipidemia in market workers who work in the municipal market in Guanambi/BA. Materials and Methods: This is an epidemiological cross-sectional and census study, in which sociodemographic, labor and health conditions of market workers with dyslipidemia were analyzed. Data were collected through semi-structured questionnaires, blood tests and anthropometric assessment. After being collected, the data were tabulated and analyzed using the Epi Info program, version 7.0. Results: The study had a total population of 426 workers, of which 241 were female, 199 underwent a blood test, and all of them had some type of dyslipidemia, were aged between 45 and 59 years old, incomplete elementary school, most had a steady partner. Most of them did not use alcohol or tobacco and did not practice physical activity. The vendors worked mainly in pavilion 3 , had a weekly working day of more than 44 hours and most reported not having a medical diagnosis for any disease. Conclusion: In view of the above, it is necessary for municipal bodies and institutions to develop interventionist policies to ensure adequate working conditions and educational actions in health.

Keywords: Occupational Health. Informal Workers. Women's Health.

Submetido em: 08 de nov. 2019

Aceito em: 14 de fev. 2020

${ }^{1}$ Universidade do Estado da Bahia - UNEB, Guanambi, Bahia - Brasil

*E-mail para correspondência: grasisantos214@gmail.com

Rev. ComCiência - jun. 2020, vol. 5, no. 6, p. 31-35 / doi: 10.36112/ issn2595-1890.v5.i6.p31-35 
Dentre os âmbitos da economia informal pode-se destacar a feira livre, na qual o seu envoltório permeia-se por dificuldades relacionadas à jornada de trabalho extensa, condições laborais inadequadas, infraestrutura deficiente, carência de horário pré-estabelecido para a realização de refeições e percalços econômicos em virtude da variação nos lucros obtidos devido ao caráter autônomo da profissão (MAGALHÃES et al., 2016).

Dentre os âmbitos da economia informal pode-se destacar a feira livre, na qual o seu envoltório permeia-se por dificuldades relacionadas à jornada de trabalho extensa, condições laborais inadequadas, infraestrutura deficiente, carência de horário pré-estabelecido para a realização de refeições e percalços econômicos em virtude da variação nos lucros obtidos devido ao caráter autônomo da profissão (MAGALHÃES et al., 2016).

Neste cenário, por representar um grupo vulnerável ao adoecimento em virtude da exposição aos riscos laborais, as mulheres feirantes necessitam de cuidados embasados em suas singularidades. Dentre as complicações advindas do trabalho informal pode-se citar as dislipidemias.

Estas complicações são caracterizadas pelos níveis elevados de lipoproteína de baixa densidade (LDL-C) e de triglicerídeos (TG), baixas concentrações de lipoproteína de alta densidade (HDL-C), ou mesmo suas combinações (FALUDI et al., 2017).Tendo ainda associação com trabalho informal, sedentarismo, hábitos alimentares e idade avançada, o que pode impactar no risco de patologias cardiovasculares nas trabalhadoras (PEREIRA et al., 2015).

Com base no exposto, a presente pesquisa tem por objetivo descrever a prevalência de dislipidemias em trabalhadoras feirantes que atuam no mercado municipal de Guanambi/BA.

\section{MÉTODOS}

Trata-se de um estudo epidemiológico de corte transversal e censitário, que foi desenvolvido a partir de dados do projeto de pesquisa intitulado "Acidentes de trabalho em feirantes e as condições laborais e de saúde: estudo prospectivo", abarcando os dados sociodemográficos, laborais e condições de saúde de trabalhadores feirantes de um mercado municipal em Guanambi/BA.

O presente estudo foi desenvolvido no Mercado Municipal da cidade de Guanambi, localizada no alto sertão do estado da Bahia. A população de estudo tratou-se de trabalhadores que desenvolviam atividades comerciais no Mercado Municipal de Guanambi, como camelôs, feirantes ou ambulantes, sem o registro de tal atividade em carteira de trabalho. Para elaboração do referido resumo, foram consideradas somente as mulheres feirantes que apresentaram algum tipo de dislipidemia.

Com a aprovação do projeto de pesquisa por parte do CEP UNEB a coleta de dados iniciou-se a partir da realização de um estudo piloto, em janeiro e fevereiro de 2018, para testar os instrumentos de coleta.

Após a aplicação do estudo piloto, procedeu-se com a etapa base (baseline), por meio de aplicação de formulário a todos os trabalhadores selecionados e que aceitaram participar do estudo, bem como realização de coleta sanguínea, medições de níveis da pressão arterial e de dados antropométricos. Ressalta-se que os dados deste estudo foram coletados na baseline.
Para construção deste estudo as seguintes variáveis foram utilizadas: características sociodemográficas (sexo, idade, convive maritalmente e grau de instrução), ocupacionais (tipo de jornada de trabalho e local de trabalho), hábitos de vida (realização de atividade física, fumar, beber), existência de morbidades e níveis lipídicos (triglicerídeos, LDL, HDL).

Os dados referentes aos níveis lipídicos foram classificados a partir dos parâmetros adotados pela Atualização da Diretriz Brasileira Sobre Dislipidemias e Prevenção da Aterosclerose - 2017 (FALUDIet al., 2017): elevação isolada do LDL-C ( $\geq 160$ $\mathrm{mg} / \mathrm{dL})$; elevação isolada dos TG ( $\geq 150 \mathrm{mg} / \mathrm{dL})$; valores aumentados de ambos LDL-C ( $\geq 160 \mathrm{mg} / \mathrm{dL})$ e TG ( $\geq 150 \mathrm{mg} / \mathrm{dL})$; redução do HDL-C (homens <40 mg/dL e mulheres <50 $\mathrm{mg} / \mathrm{dL}$ ) isolada ou em associação com aumento de LDL-C ou de TG, sendo considerado dislipidêmico aquele que possuía ao menos uma das alterações acima referidas.

Após a coleta de dados, os mesmos foram tabulados e analisados por meio Epi Info versão 7.0, no qual foram analisadas às frequências relativas e absolutas para as seguintes variáveis.

O estudo foi submetido, apreciado e aprovado pelo Comitê de Ética em Pesquisa da UNEB, sob CAAE número 77090717.8.0000.0057, sendo respeitados todos os preceitos éticos das pesquisas com seres humanos citados na Resolução 466 do Conselho Nacional de Saúde.

\section{RESULTADOS E DISCUSSÃO}

$\mathrm{O}$ presente estudo contou com uma população total de 426 trabalhadores, destes $399(93,7 \%)$ realizaram 
coleta sanguínea, enquanto 27 (6,3\%) não realizaram.

Dentre os 399 trabalhadores que realizaram exame de sangue, 241 $(60,4 \%)$ eram do sexo feminino. Destas, $199(82,6 \%)$ realizaram coleta de sangue, sendo que $194(80,5 \%)$ realizaram uma avaliação completado LDL, HDL, triglicerídeos (TG) e do triglicerídeo/LDL, 5 mulheres $(2,1 \%)$ realizaram a avaliação apenas do HDL e triglicerídeo enquanto 42 trabalhadoras $(17,4 \%)$ não realizaram o exame de sangue.

Das 399 trabalhadoras que realizaram exame de sangue, 118 (59,3\%) apresentaram níveis normais de TG, enquanto $81 \quad(40,7 \%)$ apresentaram elevação do mesmo.No que se refere a avaliação do HDL-C, 21 feirantes $(10,5 \%)$ não apresentaram redução dos valores e $178(89,5 \%)$ registraram redução do mesmo. O LDL-C registrou $154(79,4 \%)$ casos com valores normais, enquanto $40(20,6 \%)$ registraram elevação dos valores. No que diz respeito à elevação de LDL e TG, das 194 feirantes avaliadas 178 (91,7) não registraram elevação conjunta de ambos os valores, enquanto $16(8,3 \%)$ registraram elevação em ambos.

Das mulheres que realizaram coleta sanguínea, todas apresentaram algum tipo de dislipidemia. Ressalta-se que neste estudo foi considerado dislipidêmico aquele que possuía ao menos uma das alterações acima referidas (TG, LDL, HDL, TG/LDL).

A partir dos dados apresentados, a dislipidemia pode ser definida como uma alteração dos níveis séricos dos lipídeos, tais modificações podem levar a um colesterol total alto, triglicerídeo (TG) alto, colesterol de lipoproteína de alta densidade baixo (HDL-c) e níveis elevados de colesterol de lipoproteína de baixa densidade (LDL-c), considerada também fator de risco para o desenvolvimento de doenças cerebrovasculares e cardiovasculares, como infarto agudo do miocárdio e o acidente vascular cerebral (FALUDI et al., 2017).

As dislipidemias podem ter diversas classificações, no entanto na prática médica as mais utilizadas são conforme a sua etiologia e classificação laboratorial. Com base na etiologia elas podem ser primárias e secundárias, as primárias têm origem por meio dos distúrbios lipídicos e influência genética e as secundárias são ocasionadas em virtude do estilo de vida inadequado, doenças e uso de medicamentos (BERTOLAMI; BERTOLAMI 2014).

Tal patologia nem sempre apresenta sinais clínicos, no entanto, a literatura traz que pode ocorrer o aparecimento de xantomas eruptivos e tuberosos, xantomas nas pálpebras, estrias de cor alaranjadas localizadas na palma das mãos, o arco corneano, causado pelo depósito de gordura ao redor da íris, alterações na retina ou até mesmo pancreatite (IZAR; FONSECA; FONSECA, 2014).

De acordo com as variáveis sociodemográficas, a faixa etária com maior prevalência de dislipidemias $(\mathrm{n}=$ $80,40,2 \%$ ) corresponde a 45-59 anos e faixa menos prevalente $(n=26,13,1 \%)$ foi $15-29$ anos. A respeito disso, a literatura traz que a idade é um fator não modificável, ela promove diversas alterações no organismo humano com o avançar da mesma, e quando relacionados a outros fatores como maus hábitos de vida pode contribuir para o agravamento ou surgimento de diversas patologias (TORRES et al., 2018).

A maioria das trabalhadoras acometidas pelas dislipidemias cursaram até $o$ fundamental incompleto $(n=88,44,2 \%)$ e o ensino médio completo $(n=51,25,6 \%)$, enquanto que 6
(3\%) cursaram o superior completo. A respeito disso, pesquisa aponta que o nível de escolaridade está relacionado ao estilo de vida do indivíduo e que o alto grau de instrução está associado a maior conhecimento que leva a escolhas mais saudáveis, como uma alimentação equilibrada e a prática de atividade física (TEIXEIRA et al., 2019).

Tabela 01 - Perfil sociodemográfico e hábitos de vida de mulheres feirantes com dislipidemias de mercado municipal do interior baiano, 2018.

\begin{tabular}{|c|c|c|c|}
\hline VARIÁVEIS & CATEGORIAS & $\mathbf{n}$ & $\%$ \\
\hline \multicolumn{4}{|c|}{ Faixa etária } \\
\hline & $15-29$ & 26 & 13,1 \\
\hline & $30-44$ & 55 & 27,6 \\
\hline & $45-59$ & 80 & 40,2 \\
\hline & 60 mais & 38 & 19,1 \\
\hline \multicolumn{4}{|c|}{ Grau de instrução } \\
\hline & Analfabeto & 4 & 2,0 \\
\hline & Alfabetizado & 22 & 11,1 \\
\hline & Fundamental incompleto & 88 & 44,2 \\
\hline & Fundamental completo & 13 & 6,5 \\
\hline & Médio incompleto & 12 & 6,0 \\
\hline & Médio completo & 51 & 25,6 \\
\hline & Superior incompleto & 2 & 1,0 \\
\hline & Superior completo & 6 & 3,0 \\
\hline & Não sabe & 1 & 0,6 \\
\hline \multicolumn{4}{|c|}{ Convívio marital } \\
\hline & Sim & 113 & 56,8 \\
\hline & Não & 86 & 43,2 \\
\hline \multicolumn{4}{|l|}{ Fuma } \\
\hline & Sim & 18 & 9,1 \\
\hline & Não & 181 & 90,9 \\
\hline \multicolumn{4}{|c|}{ Ingesta de bebida } \\
\hline & Sim & 58 & 29,1 \\
\hline & Não & 141 & 70,9 \\
\hline \multicolumn{4}{|c|}{ Atividade física } \\
\hline & Sim & 67 & 33,7 \\
\hline & Não & 132 & 66,3 \\
\hline Total & & 199 & 100 \\
\hline
\end{tabular}

No que se refere ao convívio marital, a maioria possuía um parceiro(a) $(\mathrm{n}=113,56,8 \%)$.Todavia, em virtude do ineditismo da pesquisa não foi possível comparar se as mulheres que tinham este tipo de relação conjugal eram mais acometidas por esta patologia do que as que não mantinham convívio marital, mas tal fato pode ser justificado devido à dupla jordana de trabalho, na qual as mulheres precisam trabalhar cuidar da casa, dos filhos e marido, o que pode dificultar a procura por assistência a saúde devido à falta de tempo, um fator de 
destaque a ser elencado também é o horário de funcionamento das unidades básicas, no qual as feirantes estão realizando as atividades laborativas.

Ao analisar o uso do fumo $(n=181,90,9 \%)$ e do álcool $(n=141$, $70,9 \%$ ), verifica-se que a maioria não fazia uso destes.

Sobre o tabaco, é importante ressaltar que este acentua os distúrbios lipídicos, o que acarreta a redução em graus diferentes do HDL colesterol bom, associado ao aumento do LDL e dos TG (MIRANDA et al., 2010).

No que se refere à ingesta de bebida alcoólica, é imprescindível destacar que o consumo desta é considerada fator de risco para a ocorrência de doenças crônicas não transmissíveis, bem como o tabagismo (IBGE, 2013).

Contudo, essas práticas não apresentaram influência para ocorrência de dislipidemias no presente estudo, visto que a maioria das feirantes não fazia uso do tabaco nem de bebida alcoólica, sendo assim, as mesmas apresentaram hábitos de vida saudáveis quando analisados tais fatores.

A respeito da prática de atividade física, $132(66,3 \%)$ trabalhadoras não realizavam nenhum tipo de atividade, enquanto $67(33,7 \%)$ realizavam algum tipo de exercício físico, no mínimo, duas vezes na semana. Ressaltase que a prática atividade física é de suma importância para a melhora do perfil lipídico do indivíduo, pois permite o aumento das concentrações de HDL e a redução das concentrações de LDL e TG, que por sua vez, auxilia na prevenção e controle de doenças (CAMBRI et al., 2004).

Ao verificar a situação de trabalho, observou-se que boa parte das trabalhadoras dislipidêmicas $(n=75$, $37,7 \%$ ) pertencia ao pavilhão 3 (local no mercado municipal que abriga açougues, bancas de frutas e verduras, bancas de cereais, lanchonetes e restaurantes), seguido pelas bancas ( $n=67$, $33,7 \%$ ). Em contrapartida, o pavilhão 2 (que abriga bancas de frutas e verduras, açougues, peixarias e lanchonetes), representou o local com menor número de trabalhadoras com dislipidemia $(\mathrm{n}=21,10,6 \%)$ seguido pelo pavilhão $1(\mathrm{n}=36,18 \%))$.

A respeito da variável supracitada, não foi possível provar se de fato as mulheres do pavilhão 3 , eram mais acometidas pelas dislipidemias por fatores desencadeantes da patologia (hábitos de vida, condições de trabalho, dentre outros) ou se o número elevado de casos no referido setor foi devido o mesmo possuir o maior quantitativo de trabalhadoras e por conter (dentre açougues e bancas de cereais) restaurantes, lanchonetes e bancas de frutas e verduras, que são locais nos quais a mão de obra feminina é mais acentuada quando comparado aos outros setores.

De acordo com a variável jornada de trabalho, em horas semanais, a maior parte das feirantes $(n=112$, $56,3 \%$ ) trabalha mais de 44 horas por semana, enquanto $87(43,7 \%)$ tem uma jornada de até 44 horas. Este ainda é um fator de disparidade entre os gêneros fazendo com que muitas mulheres tenham que aderir fortemente ao setor informal o que as deixam expostas a todo e qualquer tipo de vulnerabilidade econômica, social e laboral, e isto é mais perceptível ainda com o avançar da idade (ILO, 2018).

No que diz respeito às condições de saúde das mulheres feirantes, a maioria não apresentou diagnóstico para nenhuma doença $(n=83,41,7 \%)$, todavia, $1,6 \% \quad(n=3)$ das mulheres registraram diagnóstico para cinco patologias ou mais. Mesmo equivalendo a um número pequeno de trabalha- doras, o mesmo deve ser levado em consideração, visto se tratar de um grande número de doenças em um único indivíduo. Além disso, enfatiza-se que a dislipidemia é um fator de risco para doenças cardiovasculares sendo associada ao desenvolvimento de placas ateroscleróticas, que é uma das complicações iniciais para acidente vascular cerebral e infarto agudo do miocárdio (FALUDI et al., 2017).

Tabela 02 - Condições de saúde de trabalhadoras feirantes com dislipidemias em mercado municipal de município baiano.

\begin{tabular}{clcr}
\hline VARIÁVEIS & CATEGORIAS & n & \% \\
\hline $\begin{array}{c}\text { Presença de } \\
\text { doença }\end{array}$ & & & \\
\hline & Não & 83 & 41,7 \\
& Uma doença & 56 & 28,1 \\
& Duas doenças & 38 & 19,1 \\
& Três doenças & 13 & 6,5 \\
& Quatro doenças & 6 & 3,0 \\
& Cinco ou mais doenças & 3 & 1,6 \\
\hline HAS & Sim & & \\
\hline & Não & 47 & 23,6 \\
& & 152 & 76,4 \\
\hline DM & Sim & 17 & 8,5 \\
\hline & Não & 182 & 91,5 \\
\hline Total & & $\mathbf{1 9 9}$ & $\mathbf{1 0 0}$ \\
\hline
\end{tabular}

A respeito das condições de saúde das trabalhadoras, 152 não possuíam diagnóstico para hipertensão arterial $(76,4 \%)$ e $182(91,5 \%)$ não possuíam diabetes. No entanto, $23,6 \%$ e $8,5 \%$ das feirantes apresentaram diagnóstico positivo para HAS e para DM, respectivamente.

Para tanto, a literatura apresenta que níveis elevados de colesterol no organismo humano pode promover alterações no sistema circulatório e endócrino, podendo desencadear o surgimento da HAS e do DM, e a presença desta patologia associadas à níveis lipídicos elevados se torna um fator de risco propenso as doenças cardiovasculares e cerebrovasculares (IBGE, 2014). 


\section{CONCLUSÃO}

A presente pesquisa constatou que 199 feirantes do sexo feminino apresentaram algum tipo de dislipidemia, com faixa etária entre 45 a 59 anos, com ensino fundamental incompleto, a maioria possuía um parceiro (a) fixo. A maior proporção das trabalhadoras não fazia uso de bebida alcoólica, negaram o uso de cigarro e relataram a ausência de atividade física. Acerca das condições laborais, as feirantes atuavam no pavilhão 3 e possuíam jornada de trabalho semanal com mais de 44 horas. Com relação às condições de saúde, a maior parte relatou não apresentar nenhum diagnóstico de doença.

Ressalta-se que foram encontrados alguns percalços durante a realização da coleta na presente pesquisa, como a exemplo da não participação efetiva dos feirantes nas atividades propostas, visto que os mesmos remarcavam as coletas de exames e entrevistas inúmeras vezes devido suas cargas excessivas de trabalho.

Neste contexto, medidas legislativas devem ser tomadas pelas esferas governamentais municipais, objetivando a garantia de condições trabalhistas favoráveis para esse público, bem como as instituições de ensino e saúde do município podem intervir com ações educativas e intervencionais, além disso, fazem-se necessárias melhorias de infraestrutura da feira para que as trabalhadoras possam desenvolver suas atividades laborais de forma segura.

\section{REFERÊNCIAS}

BERTOLAMI, A, BERTOLAMI, M.C. Dislipidemias / Dyslipidemias. Revista Brasileira de Medicina. v. 12, n.71, 2014. Disponível em: bireme.br/cgiin/wxislind.exe/iah/online/?Is
isS-

cript $=\mathrm{iah} / \mathrm{iah} . \mathrm{xis} \& \mathrm{src}=\mathrm{goog} 1 \% 20 \mathrm{e} \& \mathrm{bas}$ e=LILACS\&lang=p\&nextAction=lnk \&exprSearch=737096\&indexSearch=I D >. Acesso em: 10 out 2019.

CAMBRI, L. T. et al. Perfil lipídico, dislipidemia e exercícios físicos. Revista Brasileira de Cinantropometria e Desempenho Humano. v. 8, n. 3, p. 100-106, 2006. Disponível em:

< https://periodicos.ufsc.br/index.php/r bcdh/article/view/3916/16575>. Acesso em: 10 out 2019.

FALUDI, A. A. et al. Atualização daDiretriz Brasileira de Dislipidemias e Prevenção da Aterosclerose - 2017. Arq. Bras. Cardiol, 2017. Disponível em:

https://doi.org/10.5935/abc.20170121

\&62: Acessoem: 08 maio. 2019.

INSTITUTO BRASILEIRO DE GEOGRAFIA E ESTATÍTICA. Pesquisa Nacional de Saúde 2013: percepção do estado de saúde, estilos de vida e doenças crônicas. Brasil, grandes regiões e unidades da federação. Rio de Janeiro: IBGE, 2003. p. 180, 2014.

ILO. International Labour Office.

Women and men in the informal economy: A statistical picture, 2018. Disponível em: <

https://www.ilo.org/wcmsp5/groups/pu blic/---dgreports/---

dcomm/documents/publication/wcms_ 626831.pdf> Acesso em: 07 out 2019.

IZAR, M. C.; FONSECA, M. I. H.; FONSECA, F. A. H. Dislipidemias. Revista Brasileira Medicina. v. 8, n.71, 2014. Disponível

em:http://www.moreirajr.com.br/revist as.asp?fase=r003\&id_materia $=4566>$. Acesso em:10 out 2019.

MAGALHÃES, A.H.R. et al. Necessidade de saúde das mulheres feirantes: acesso, vínculo e acolhimento como práticas de integralidade. Revista Gaúcha de Enfermagem. v 37, (esp), p. 2016-2026, 2016. Disponível em:<http://www.scielo.br/scielo.php?s cript $=$ sci_arttext\&pid=S1983-
$14472016000500417 \& \operatorname{lng}=$ en $\& n r m=$ is o>. Acesso em:10 out 2019.

MIRANDA, T. V. et al. Análise dos fatores associados à dislipidemia. Revista Brasileira de Medicina. v. 24, p. 3-14, 2010. Disponível em: http://files.bvs.br/upload/S/01015907/2010/v24n3-4/a2342.pdf > . Acesso em:10 out 2019.

PEREIRA, L. P.et al. Dislipidemia autorreferida na região Centro-Oeste do Brasil: prevalência e fatores associados.Ciência \& Saúde Coletiva, v. 20, n. 6, p. 1815-1824, 2015. Disponível em:

$<$ http://www.scielo.br/scielo.php?scrip $\mathrm{t}=$ sci_arttext\&pid=S 1413-

$81232015000601815 \& \operatorname{lng}=\mathrm{en} \& \mathrm{nrm}=$ is o>. Acesso em:10 out 2019.

TORRES, F. D. G. et al. Prevalência da síndrome metabólica na população de 15 a 74 anos de idade no município de Guantánamo. Revista de informação científica. v 97, n. 5, p. 987-998 set.-out. 2018. Disponível em: <http://www.revinfcientifica.sld.cu/ind ex.php/ric/article/view/2147/3911>. Acesso em: 10 out 2019. 